\title{
$h p$-Discontinuous Galerkin Finite Element Methods for Hyperbolic Problems: Error Analysis and Adaptivity
}

\author{
Endre Süli \\ University of Oxford, Computing Laboratory \\ Paul Houston Bill Senior \\ Department of Mathematics \& Computer Science, University of Leicester
}

\section{Introduction}

Hyperbolic partial differential equations arise in the mathematical modelling of many physical phenomena and their accurate numerical solution is of great practical importance. The present paper is concerned with the question of a posteriori error estimation and the construction of adaptive algorithms that are capable of delivering numerical approximations to the unknown analytical solution of a hyperbolic problem, accurate to within a user-prescribed tolerance. Although we shall focus on one particular class of numerical techniques, the discontinuous Galerkin finite element method (DGFEM), the implications of the analysis presented here are wider, and the ideas developed apply more generally.

Adaptive finite element methods which admit both local polynomial-degree-variation $(p-$ refinement) and local mesh subdivision ( $h$-refinement) offer greater flexibility and improved efficiency than mesh refinement methods which rely on $h$-refinement or $p$-refinement in isolation. The aim of this paper is, therefore, to develop the a posteriori error analysis of the $h p$-version of the DGFEM for hyperbolic problems; see [4] and the references therein, and [3] for earlier work in this area. In particular, we shall be concerned with the derivation of computable error bounds for linear functionals of the solution: physically relevant examples include problems of lift and drag computation, the calculation of the mean value of the field over the computational domain or its normal flux through the boundary.

The approach pursued here rests on a hyperbolic duality argument. In order to highlight the key ideas and put them into a general context, we begin in Section 2 with an overview of adjoint methods and duality. In Section 3 we introduce the model problem and formulate the DGFEM. Then, in Section 4, we derive a posteriori error bounds for linear functionals of the solution; the bounds involve computable residual terms multiplied by local weights involving the dual solution, cf. [6,9]. Guided by our a posteriori error analysis, we then design an $h p$-adaptive finite element algorithm to guarantee both reliable and efficient control of the error in the approximation to the functional with respect to a fixed user-defined tolerance.

\section{Adjoint methods and duality}

The question we wish to explore can be, briefly, formulated as follows. Suppose that $X$ and $Y$ are two Hilbert spaces, $B(\cdot, \cdot)$ is a bounded bilinear functional defined on $X \times Y$, namely

$$
|B(w, v)| \leq c_{1}\|w\|_{X}\|v\|_{Y} \quad \forall w \in X \quad \forall v \in Y,
$$

where $c_{1}$ is a positive constant, and $\ell$ is a bounded linear functional defined on $Y$; suppose that $u$ is the unique solution to the variational problem

$$
\text { find } u \in X \text { such that } B(u, v)=\ell(v) \quad \forall v \in Y \text {. }
$$


Problem $(P)$ can be thought of as the weak formulation of a linear partial differential equation on $X$ whose unique solution is $u \in X$. It is frequently the case in applications that, instead of $u$, the focus of interest is a derived quantity $J(u)$ which should be computed to within a given tolerance TOL, where $J$ is a given bounded linear functional defined on $X$. Indeed, it may happen that instead of a single linear functional $\ell$, several linear functionals $\ell_{i}, i=1, \ldots, N$, are given, and instead of a single quantity $J(u)$ one needs to compute $J\left(u_{i}\right), i=1, \ldots, N$, each to within a given tolerance TOL, where $u_{i}$ is the solution to the problem:

$$
\text { find } u_{i} \in X \text { such that } B\left(u_{i}, v\right)=\ell_{i}(v) \quad \forall v \in Y \text {, }
$$

for $i=1, \ldots, N$. Problems of this kind arise in engineering design when the linear functional $\ell$ depends on a design variable $\omega$, i.e., $\ell(v)=\ell(v ; \omega)$, and $(P)$ has to be solved for an entire set $\omega \in\left\{\omega_{1}, \ldots, \omega_{N}\right\}$, leading to a sequence of problems $\left(P_{i}\right)$ with $\ell_{i}(v)=\ell\left(v ; \omega_{i}\right), i=1, \ldots, N$.

The Direct Method for computing $J\left(u_{i}\right), i=1, \ldots, N$, is to solve problem $\left(P_{i}\right)$ for $u_{i}$ and evaluate $J\left(u_{i}\right)$ for each $i=1, \ldots, N$. Upon discretisation, this amounts to solving $N$ systems of linear equations with the same matrix but $N$ different right-hand-side vectors.

The alternative approach, referred to as the Adjoint Method, is based on considering the following dual problem:

$$
\text { find } z \in Y \text { such that } B(w, z)=J(w) \quad \forall w \in X .
$$

A key observation is the following primal-dual equivalence relating $(P)$ to $(D)$ :

$$
J(u)=B(u, z)=\ell(z) .
$$

When applied to $\left(P_{i}\right)$ and $(D)$, identity $(2.1)$ yields

$$
J\left(u_{i}\right)=B\left(u_{i}, z\right)=\ell_{i}(z), \quad i=1, \ldots, N .
$$

The practical implication of (2.2) is that instead of solving $N$ copies of the primal problem $(P)$ for $u_{i} \in X$ with data $\ell_{i}, i=1 \ldots, N$, and then evaluate $J\left(u_{i}\right)$ for $i=1, \ldots, N$, one can, instead, simply solve problem $(D)$ for $z \in Y$ and evaluate $\ell_{i}(z)$ for $i=1, \ldots, N$. When $N \gg 1$ the Adjoint Method is, computationally, much more attractive than the Direct Method.

Of course, in practice, problems $(P)$ and $(D)$ cannot, in general, be solved in closed form but need to be approximated numerically. Suppose therefore that $\left\{X_{h}\right\}$ is a sequence of finite-dimensional spaces contained in $X$ and $\left\{Y_{h}\right\}$ a sequence of finite-dimensional spaces contained in $Y$. We approximate $(P)$ by the following problem:

$$
\text { find } u_{h} \in X_{h} \text { such that } B\left(u_{h}, v_{h}\right)=\ell\left(v_{h}\right) \quad \forall v_{h} \in Y_{h} \text {. }
$$

The fundamental question that arises then is how well $J(u)$ is approximated by $J\left(u_{h}\right)$. We shall use a duality argument for the quantitative assessment of the approximation error.

Remark 1 In analogy with $(P)$, Problem $\left(P_{i}\right)$ can be discretised as follows: for $i=1, \ldots, N$,

$$
\text { find } u_{i h} \in X_{h} \text { such that } B\left(u_{i h}, v_{h}\right)=\ell_{i}\left(v_{h}\right) \quad \forall v_{h} \in Y_{h} \quad\left(P_{i h}\right) \text {. }
$$

Given that $(P)$ and $(D)$ are driven by different data, the sequence of test spaces $\left\{Y_{h}\right\}$ used in the discretisation of the primal problem need not be an adequate sequence of trial spaces for the numerical approximation of the dual solution $z \in Y$; a similar comment applies to the sequence of primal trial spaces $\left\{X_{h}\right\}$. Thus we select two additional families of finitedimensional spaces $\left\{X_{H}\right\}$, with $X_{H} \subset X$ and $\left\{Y_{H}\right\}$, with $Y_{H} \subset Y$, and consider the following discretisation of the dual problem $(D)$ :

$$
\text { find } z_{H} \in Y_{H} \text { such that } B\left(w_{H}, z_{H}\right)=J\left(w_{H}\right) \quad \forall w_{H} \in X_{H} \text {. }
$$


The discrete counterpart of the primal-dual equivalence (2.1) is

$$
J\left(u_{h}\right)=\ell\left(z_{H}\right)+\rho_{h H},
$$

where, $\rho_{h H}=B\left(u-u_{H}, z-z_{H}\right)-B\left(u-u_{h}, z-z_{h}\right)$ for all $u_{H}$ in $X_{H}$ for all $z_{h}$ in $Y_{h}$. In particular if $X_{h} \subset X_{H}$ and $Y_{H} \subset Y_{h}$, then we can choose $u_{H}=u_{h}$ and $z_{h}=z_{H}$, yielding $\rho_{h H}=0$. In general, however, these inclusions will not hold. At any rate,

$$
\begin{aligned}
\left|J\left(u_{h}\right)-\ell\left(z_{H}\right)\right| & \leq \inf _{u_{H} \in X_{H}} \inf _{z_{h} \in Y_{h}}\left|B\left(u-u_{H}, z-z_{H}\right)-B\left(u-u_{h}, z-z_{h}\right)\right| \\
& \leq \inf _{u_{H} \in X_{H}}\left|B\left(u-u_{H}, z-z_{H}\right)\right|+\inf _{z_{h} \in Y_{h}}\left|B\left(u-u_{h}, z-z_{h}\right)\right| \\
& \leq c_{1} \inf _{u_{H} \in X_{H}}\left\|u-u_{H}\right\|_{X}\left\|z-z_{H}\right\|_{Y}+c_{1}\left\|u-u_{h}\right\|_{X} \inf _{z_{h} \in Y_{h}}\left\|z-z_{h}\right\|_{Y} .
\end{aligned}
$$

Consequently, $\ell\left(z_{H}\right)$ is an "order-doubling" approximation to $J\left(u_{h}\right)$ in the following sense.

Theorem 2 Suppose that the bilinear form $B(\cdot, \cdot)$ is weakly coercive on $X_{h} \times Y_{h}$; namely,

(a) There exists a constant $c_{0}>0$ such that

$$
\inf _{w_{h} \in X_{h} \backslash\{0\}} \sup _{v_{h} \in Y_{h} \backslash\{0\}} \frac{\left|B\left(w_{h}, v_{h}\right)\right|}{\left\|w_{h}\right\|_{X}\left\|v_{h}\right\|_{Y}} \geq c_{0} ;
$$

(b) $\sup _{w_{h} \in X_{h}} B\left(w_{h}, v_{h}\right)>0$ for all $v_{h} \in Y_{h} \backslash\{0\}$.

Then problem $\left(P_{h}\right)$ has a unique solution $u_{h} \in U_{h}$, and

$$
\left\|u-u_{h}\right\|_{X} \leq C_{p} \inf _{w_{h} \in X_{h}}\left\|u-w_{h}\right\|_{X}
$$

where $C_{p}=1+\left(c_{1} / c_{0}\right)$.

If $B(\cdot, \cdot)$ is weakly coercive on $X_{H} \times Y_{H}$ then $\left(D_{H}\right)$ has a unique solution $z_{H} \in Y_{H}$ and

$$
\left\|z-z_{H}\right\|_{Y} \leq C_{d} \inf _{v_{H} \in Y_{H}}\left\|z-v_{H}\right\|_{Y}
$$

where $C_{d}=C_{d}\left(c_{0}, c_{1}\right)$ is a positive constant.

If $B(\cdot, \cdot)$ is weakly coercive on both $X_{h} \times Y_{h}$ and $X_{H} \times Y_{H}$, then

$$
\begin{aligned}
\left|J\left(u_{h}\right)-\ell\left(z_{H}\right)\right| \leq & c_{1}\left(C_{p} \inf _{w_{h} \in X_{h}}\left\|u-w_{h}\right\|_{X} \inf _{v_{h} \in Y_{h}}\left\|z-v_{h}\right\|_{Y}\right. \\
& \left.+C_{d} \inf _{w_{H} \in X_{H}}\left\|u-w_{H}\right\|_{X} \inf _{v_{H} \in Y_{H}}\left\|z-v_{H}\right\|_{Y}\right) .
\end{aligned}
$$

Now assuming that the spaces $X_{h}, Y_{h}, X_{H}$ and $Y_{H}$ possess suitable approximation properties, it is possible to derive an a priori error bound on $\left|J\left(u_{h}\right)-\ell\left(z_{h}\right)\right|$ in terms of the discretisation parameters $h$ and $H$ and norms of the primal and dual solutions $u$ and $z$.

A further, and practically more significant, requirement is to quantify the error between the unknown value of $J(u)$ and its computed approximation $J\left(u_{h}\right)$; more specifically, we consider the following measurement problem: find $J\left(u_{h}\right) \in \mathbb{R}$ such that

$$
\left|J(u)-J\left(u_{h}\right)\right| \leq \mathrm{TOL},
$$

where TOL is a (given) positive tolerance. Our approach to the solution of this problem again rests on a duality argument. We begin by noting that, by $(D),(P)$ and $\left(P_{h}\right)$,

$$
J(u)-J\left(u_{h}\right)=J\left(u-u_{h}\right)=B\left(u-u_{h}, z\right)=B\left(u-u_{h}, z-z_{h}\right) \quad \forall z_{h} \in Y_{h} .
$$


Further, by $(P)$ again,

$$
B\left(u-u_{h}, z-z_{h}\right)=B\left(u, z-z_{h}\right)-B\left(u_{h}, z-z_{h}\right)=\ell\left(z-z_{h}\right)-B\left(u_{h}, z-z_{h}\right) .
$$

As $v \mapsto \ell(v)-B\left(u_{h}, v\right)$ is a bounded linear functional on $Y$ for each $u_{h} \in X_{h}$, there exists $R_{p}\left(u_{h}\right) \in Y^{\prime}$ (where $Y^{\prime}$ denotes the dual space of $Y$ ) such that

$$
\ell\left(z-z_{h}\right)-B\left(u_{h}, z-z_{h}\right)=\left\langle R_{p}\left(u_{h}\right), z-z_{h}\right\rangle
$$

here $\langle\cdot, \cdot\rangle$ denotes the duality pairing between $Y^{\prime}$ and $Y$. The element $R_{p}\left(u_{h}\right)$ will be referred to as the primal residual. Combining the last three identities, we arrive at the following primal error representation formula

$$
J(u)-J\left(u_{h}\right)=\left\langle R_{p}\left(u_{h}\right), z-z_{h}\right\rangle \quad \forall z_{h} \in Y_{h}
$$

which will be the starting point of our a posteriori error analysis.

Finally, we note that an analogous argument leads to the dual error representation formula

$$
\ell(z)-\ell\left(z_{h}\right)=\left\langle u-u_{H}, R_{d}\left(z_{H}\right)\right\rangle \quad \forall u_{H} \in X_{H}
$$

where $R_{d}\left(z_{H}\right) \in X^{\prime}$ denotes the denotes the dual residual, defined as the Riesz representer of the bounded linear functional $w \mapsto J(w)-B\left(w, z_{H}\right)$; here, $\langle\cdot, \cdot\rangle$ denotes the duality pairing between $X$ and its dual space $X^{\prime}$. The dual error representation formula can now be used to solve the dual measurement problem: given a positive tolerance TOL, find $\ell\left(z_{h}\right) \in \mathbb{R}$ such that

$$
\left|\ell(z)-\ell\left(z_{h}\right)\right| \leq \text { TOL } .
$$

By the primal-dual equivalence, $\ell(z)=J(u)$; hence $J(u)$ in $(2.4)$ and $(2.5)$ can be replaced by $\ell(z)$, and $\ell(z)$ in (2.7) and (2.6) can be replaced by $J(u)$; however, as noted above, $J\left(u_{h}\right)$ and $\ell\left(z_{h}\right)$ are not interchangeable, unless $X_{h} \subset X_{H}$ and $Y_{H} \subset Y_{h}$; thus, in general, neither of (2.4) and (2.7) implies the other.

\section{The model problem and its $h p$-DGFEM approximation}

Let $\Omega$ be a bounded open polyhedral domain in $\mathbb{R}^{d}, d \geq 2$, and let $\Gamma$ denote the union of open faces of $\Omega$. Suppose that $\mathbf{B}=\left(\mathbf{B}_{1}, \ldots, \mathbf{B}_{d}\right)$ is a $d$-component matrix function defined on $\bar{\Omega}$ with $\mathbf{B}_{i} \in\left[W_{\infty}^{1}(\Omega)\right]_{\text {symm }}^{m \times m}, i=1, \ldots, d$. Let $\boldsymbol{\mu}=\left(\mu_{1}, \ldots, \mu_{d}\right)$ denote the unit outward normal vector to $\Gamma$, and consider the symmetric matrix

$$
\mathbf{B}(\boldsymbol{\mu}) \equiv \mathbf{B} \cdot \boldsymbol{\mu}=\boldsymbol{\mu}_{1} \mathbf{B}_{1}+\ldots+\boldsymbol{\mu}_{d} \mathbf{B}_{d}
$$

Suppose that $\mathbf{B}$ is diagonalised via $\mathbf{B}(\boldsymbol{\mu})=X(\boldsymbol{\mu})^{-1} \Lambda(\boldsymbol{\mu}) X(\boldsymbol{\mu})$, where $\Lambda(\boldsymbol{\mu})$ is a diagonal matrix, with the (real) eigenvalues of $\mathbf{B}(\boldsymbol{\mu})$ appearing along its diagonal. We shall suppose that $\Gamma$ is nowhere characteristic in the sense that none of the diagonal entries of $\Lambda(\boldsymbol{\mu})$ is zero for any choice of the unit outward normal vector $\boldsymbol{\mu}$ on $\Gamma$. We decompose $\Lambda(\boldsymbol{\mu})$ as

$$
\Lambda(\boldsymbol{\mu})=\Lambda_{-}(\boldsymbol{\mu})+\Lambda_{+}(\boldsymbol{\mu}),
$$

where $\Lambda_{-}(\boldsymbol{\mu})$ is diagonal and negative semidefinite, and $\Lambda_{+}(\boldsymbol{\mu})$ is diagonal and positive semidefinite. Thus we define

$$
\mathbf{B}_{-}(\boldsymbol{\mu})=X(\boldsymbol{\mu})^{-1} \Lambda_{-}(\boldsymbol{\mu}) X(\boldsymbol{\mu}) \quad \text { and } \quad \mathbf{B}_{+}(\boldsymbol{\mu})=X(\boldsymbol{\mu})^{-1} \Lambda_{+}(\boldsymbol{\mu}) X(\boldsymbol{\mu}) .
$$


Clearly, for each unit outward normal vector $\boldsymbol{\mu}$ on $\Gamma$,

$$
\mathbf{B}(\boldsymbol{\mu})=\mathbf{B}_{-}(\boldsymbol{\mu})+\mathbf{B}_{+}(\boldsymbol{\mu}) .
$$

Given $\mathbf{C} \in\left[L_{\infty}(\Omega)\right]^{m \times m}, \mathbf{f} \in\left[L_{2}(\Omega)\right]^{m}$ and $\mathbf{g} \in\left[L_{2}(\Gamma)\right]^{m}$, we consider the following hyperbolic boundary value problem: find $\mathbf{u} \in H(\mathcal{L}, \Omega)$ such that

$$
\mathcal{L} \mathbf{u} \equiv \nabla \cdot(\mathbf{B u})+\mathbf{C u}=\mathbf{f} \quad \text { in } \Omega, \quad \mathbf{B}_{-}(\boldsymbol{\mu}) \mathbf{u}=\mathbf{B}_{-}(\boldsymbol{\mu}) \mathbf{g} \quad \text { on } \Gamma
$$

where $H(\mathcal{L}, \Omega)=\left\{\mathbf{v} \in\left[L_{2}(\Omega)\right]^{m}: \mathcal{L} \mathbf{v} \in\left[L_{2}(\Omega)\right]^{m}\right\}$ denotes the graph space of the partial differential operator $\mathcal{L}$ in $L_{2}(\Omega)$. Next, we formulate the $h p$-version of the Discontinuous Galerkin Finite Element Method ( $h p$-DGFEM, for short) for the numerical solution of (3.1).

Suppose that $\mathcal{T}_{h}$ is a regular or 1-irregular subdivision of $\Omega$ into disjoint open element domains $\kappa$ such that $\bar{\Omega}=\cup_{\kappa \in \mathcal{T}_{h}} \bar{\kappa}$. Thus a $(d-1)$-dimensional face of each element $\kappa$ in $\mathcal{T}_{h}$ is allowed to contain at most one hanging (irregular) node - typically the barycenter of the face. We shall suppose that the family of subdivisions $\mathcal{T}_{h}$ is shape-regular and that each $\kappa \in \mathcal{T}_{h}$ is a smooth bijective image of a fixed master element $\hat{\kappa}$, that is, $\kappa=F_{\kappa}(\hat{\kappa})$ for all $\kappa \in \mathcal{T}_{h}$, where $\hat{\kappa}$ is either the open unit simplex or the open unit hypercube in $\mathbb{R}^{d}$. On the reference element $\hat{\kappa}$, with $\hat{\mathbf{x}}=\left(\hat{x}_{1}, \ldots, \hat{x}_{d}\right) \in \hat{\kappa}$ and $\boldsymbol{\alpha}=\left(\alpha_{1}, \ldots, \alpha_{d}\right) \in \mathbb{N}_{0}^{d}$, we define spaces of polynomials of degree $p \geq 1$ as follows:

$$
\mathcal{Q}_{p}=\operatorname{span}\left\{\hat{\mathbf{x}}^{\boldsymbol{\alpha}}: 0 \leq \alpha_{i} \leq p, \quad 1 \leq i \leq d\right\}, \quad \mathcal{P}_{p}=\operatorname{span}\left\{\hat{\mathbf{x}}^{\boldsymbol{\alpha}}: 0 \leq|\alpha| \leq p\right\} .
$$

To each $\kappa \in \mathcal{T}_{h}$ we assign an integer $p_{\kappa} \geq 1$; collecting the $p_{\kappa}$ and $F_{\kappa}$ in the vectors $\mathbf{p}=\left\{p_{\kappa}\right.$ : $\left.\kappa \in \mathcal{T}_{h}\right\}$ and $\mathbf{F}=\left\{F_{\kappa}: \kappa \in \mathcal{T}_{h}\right\}$, respectively, we introduce the finite element space

$S^{\mathbf{p}}\left(\Omega, \mathcal{T}_{h}, \mathbf{F}\right)=\left\{\mathbf{v} \in\left[L_{2}(\Omega)\right]^{m}:\left.\mathbf{v}\right|_{\kappa} \circ F_{\kappa} \in\left[\mathcal{Q}_{p_{\kappa}}\right]^{m}\right.$ if $F_{\kappa}^{-1}(\kappa)$ is the open unit hypercube, and $\left.\mathbf{v}\right|_{\kappa} \circ F_{\kappa} \in\left[\mathcal{P}_{p_{\kappa}}\right]^{m}$ if $F_{\kappa}^{-1}(\kappa)$ is the open unit simplex; $\left.\kappa \in \mathcal{T}_{h}\right\}$.

Assuming that $\mathcal{T}_{h}$ is a subdivision of $\Omega$, we consider the broken Sobolev space $H^{\mathrm{s}}\left(\Omega, \mathcal{T}_{h}\right)$ of composite index $\mathbf{s}$ with nonnegative components $s_{\kappa}, \kappa \in \mathcal{T}_{h}$, defined by

$$
\left[H^{\mathbf{s}}\left(\Omega, \mathcal{T}_{h}\right)\right]^{m}=\left\{\mathbf{v} \in\left[L_{2}(\Omega)\right]^{m}:\left.\mathbf{v}\right|_{\kappa} \in\left[H^{s_{\kappa}}(\kappa)\right]^{m} \quad \forall \kappa \in \mathcal{T}_{h}\right\}
$$

If $s_{\kappa}=s \geq 0$ for all $\kappa \in \mathcal{T}_{h}$, we shall simply write $\left[H^{s}\left(\Omega, \mathcal{T}_{h}\right)\right]^{m}$.

Given that $\kappa$ is an element in the subdivision $\mathcal{T}_{h}$, we denote by $\partial \kappa$ the union of $(d-1)$ dimensional open faces of $\kappa$. Let $\mathbf{x} \in \partial \kappa$ and suppose that $\mathbf{n}_{\kappa}(\mathbf{x})$ denotes the unit outward normal vector to $\partial \kappa$ at $\mathbf{x}$. With these conventions, we define $\mathbf{B}\left(\mathbf{n}_{\kappa}\right), \mathbf{B}_{-}\left(\mathbf{n}_{\kappa}\right)$ and $\mathbf{B}_{+}\left(\mathbf{n}_{\kappa}\right)$ analogously to $\mathbf{B}(\boldsymbol{\mu}), \mathbf{B}_{-}(\boldsymbol{\mu})$ and $\mathbf{B}_{+}(\boldsymbol{\mu})$ above, respectively. For each $\kappa \in \mathcal{T}_{h}$ and any $\mathbf{v} \in\left[H^{1}(\kappa)\right]^{m}$ we denote by $\mathbf{v}_{\kappa}^{+}$the interior trace of $\mathbf{v}$ on $\partial \kappa$ (the trace taken from within $\kappa$ ). Now consider an element $\kappa$ such that the set $\partial \kappa \backslash \Gamma$ is nonempty; then for each $\mathbf{x} \in \partial \kappa \backslash \Gamma$ (with the exception of a set of $(d-1)$-dimensional measure zero) there exists a unique element $\kappa^{\prime}$, depending on the choice of $\mathbf{x}$, such that $\mathbf{x} \in \partial \kappa^{\prime}$. Suppose that $\mathbf{v} \in\left[H^{1}(\Omega, \mathcal{T})\right]^{m}$. If $\partial \kappa \backslash \Gamma$ is nonempty for some $\kappa \in \mathcal{T}_{h}$, then we define the outer trace $\mathbf{v}_{\kappa}^{-}$of $\mathbf{v}$ on $\partial \kappa \backslash \Gamma$ relative to $\kappa$ as the inner trace $\mathbf{v}_{\kappa^{\prime}}^{+}$relative to those elements $\kappa^{\prime}$ for which $\partial \kappa^{\prime}$ has intersection with $\partial \kappa \backslash \Gamma$ of positive $(d-1)$-dimensional measure. Since below it will always be clear from the context which element $\kappa$ in the subdivision $\mathcal{T}_{h}$ the quantities $\mathbf{n}_{\kappa}, \mathbf{v}_{\kappa}^{+}$and $\mathbf{v}_{\kappa}^{-}$correspond to, for the sake of notational simplicity we shall suppress the letter $\kappa$ in the subscript and write, respectively, $\mathbf{n}, \mathbf{v}^{+}$, and $\mathbf{v}^{-}$instead. 
For $\mathbf{v}, \mathbf{w} \in\left[H^{1}\left(\Omega, \mathcal{T}_{h}\right)\right]^{m}$, we introduce the bilinear form

$$
\begin{aligned}
B_{\mathrm{DG}}(\mathbf{w}, \mathbf{v})= & \sum_{\kappa \in \mathcal{T}_{h}} \int_{\kappa} \mathbf{w} \cdot \mathcal{L}^{*} \mathbf{v} \mathrm{d} x+\sum_{\kappa \in \mathcal{T}_{h}} \int_{\partial \kappa \backslash \Gamma} \mathcal{H}\left(\mathbf{w}^{+}, \mathbf{w}^{-}, \mathbf{n}\right) \cdot \mathbf{v}^{+} \mathrm{d} s \\
& +\sum_{\kappa \in \mathcal{T}_{h}} \int_{\partial \kappa \cap \Gamma} \mathbf{B}_{+}(\mathbf{n}) \mathbf{w}^{+} \cdot \mathbf{v}^{+} \mathrm{d} s
\end{aligned}
$$

where $\mathcal{L}^{*}$ is the formal adjoint of $\mathcal{L}$ defined by $\mathcal{L}^{*} \mathbf{v} \equiv-(\mathbf{B} \cdot \nabla) \mathbf{v}+\mathbf{C}^{T} \mathbf{v} ; \mathcal{H}(\cdot, \cdot, \cdot)$ is a numerical flux function, assumed to be Lipschitz continuous, and such that:

(i) $\mathcal{H}$ is consistent; i.e., $\left.\mathcal{H}(\mathbf{u}, \mathbf{u}, \mathbf{n})\right|_{\partial \kappa \backslash \Gamma}=\left.\mathbf{B}(\mathbf{n}) \mathbf{u}\right|_{\partial \kappa \backslash \Gamma}$ for all $\kappa$ in $\mathcal{T}_{h}$;

(ii) $\mathcal{H}(\cdot, \cdot, \cdot)$ is conservative; i.e., $\left.\mathcal{H}\left(\mathbf{u}^{+}, \mathbf{u}^{-}, \mathbf{n}\right)\right|_{\partial \kappa \backslash \Gamma}=-\left.\mathcal{H}\left(\mathbf{u}^{-}, \mathbf{u}^{+},-\mathbf{n}\right)\right|_{\partial \kappa \backslash \Gamma}$.

We emphasise that the choice of the numerical flux function is completely independent of the finite element space employed. For example, one may take

$$
\mathcal{H}\left(\mathbf{u}^{+}, \mathbf{u}^{-}, \mathbf{n}\right)=\mathbf{B}_{+}(\mathbf{n}) \mathbf{u}^{+}+\mathbf{B}_{-}(\mathbf{n}) \mathbf{u}^{-}=\frac{1}{2}\left(\mathbf{B}(\mathbf{n}) \mathbf{u}^{+}+\mathbf{B}(\mathbf{n}) \mathbf{u}^{-}\right)-\frac{1}{2}|\mathbf{B}(\mathbf{n})|\left(\mathbf{u}^{-}-\mathbf{u}^{+}\right),
$$

where $|\mathbf{B}(\mathbf{n})|=\mathbf{B}_{+}(\mathbf{n})-\mathbf{B}_{-}(\mathbf{n})$. For $\mathbf{v} \in\left[H^{1}\left(\Omega, \mathcal{T}_{h}\right)\right]^{m}$, we consider the linear functional

$$
\ell_{\mathrm{DG}}(\mathbf{v})=\sum_{\kappa \in \mathcal{T}_{h}} \int_{\kappa} \mathbf{f} \cdot \mathbf{v} \mathrm{d} x-\sum_{\kappa \in \mathcal{T}_{h}} \int_{\partial \kappa \cap \Gamma} \mathbf{B}_{-}(\mathbf{n}) \mathbf{g} \cdot \mathbf{v}^{+} \mathrm{d} s .
$$

The $h p$-DGFEM for (3.1) is defined as follows: find $\mathbf{u}_{\mathrm{DG}} \in S^{\mathbf{p}}\left(\Omega, \mathcal{T}_{h}, \mathbf{F}\right)$ such that

$$
B_{\mathrm{DG}}\left(\mathbf{u}_{\mathrm{DG}}, \mathbf{v}\right)=\ell_{\mathrm{DG}}(\mathbf{v}) \quad \forall \mathbf{v} \in S^{\mathbf{p}}\left(\Omega, \mathcal{T}_{h}, \mathbf{F}\right) .
$$

\section{A posteriori error analysis}

Let us suppose that we wish to control the discretisation error in some linear functional $J(\cdot)$ defined on a linear space which contains $H(\mathcal{L}, \Omega)+S^{\mathbf{p}}\left(\Omega, \mathcal{T}_{h}, \mathbf{F}\right)$. Following the argument presented in [6] for stabilised continuous finite element approximations, we do so by deriving an a posteriori bound on the error between $J(\mathbf{u})$ and $J\left(\mathbf{u}_{\mathrm{DG}}\right)$. We begin our analysis by considering the following dual or adjoint problem: find $\mathbf{z}$ in $H\left(\mathcal{L}^{*}, \Omega\right)$ such that

$$
B_{\mathrm{DG}}(\mathbf{w}, \mathbf{z})=J(\mathbf{w}) \quad \forall \mathbf{w} \in H(\mathcal{L}, \Omega),
$$

where $H\left(\mathcal{L}^{*}, \Omega\right)$ denotes the graph space of the adjoint operator $\mathcal{L}^{*}$ in $L_{2}(\Omega)$. Let us assume that (4.1) possesses a unique solution.

Our a posteriori bound on the error between $J(\mathbf{u})$ and $J\left(\mathbf{u}_{\mathrm{DG}}\right)$ will be expressed in terms of the finite element residual $\mathbf{r}_{h, p}$ defined on $\kappa \in \mathcal{T}_{h}$ by $\left.\mathbf{r}_{h, p}\right|_{\kappa}=\left.\left(\mathbf{f}-\mathcal{L} \mathbf{u}_{\mathrm{DG}}\right)\right|_{\kappa}$, which measures the extent to which $\mathbf{u}_{\mathrm{DG}}$ fails to satisfy the differential equation on the union of the elements $\kappa$ in the mesh $\mathcal{T}_{h}$; thus we refer to $\mathbf{r}_{h, p}$ as the internal residual. Also, since $\mathbf{u}_{\mathrm{DG}}$ only satisfies the boundary conditions approximately, the vector $\mathbf{B}_{-}(\boldsymbol{\mu})\left(\mathbf{g}-\mathbf{u}_{\mathrm{DG}}\right)$ need not be zero on $\Gamma$; thus for each element $\kappa$ with $\partial \kappa \cap \Gamma$ nonempty, we define the boundary residual $\boldsymbol{\rho}_{h, p}$ by $\left.\boldsymbol{\rho}_{h, p}\right|_{\partial \kappa \cap \Gamma}=\left.\mathbf{B}_{-}(\mathbf{n})\left(\mathbf{u}_{\mathrm{DG}}^{+}-\mathbf{g}\right)\right|_{\partial \kappa \cap \Gamma}$. Analogously, on $\partial \kappa \backslash \Gamma$, we define the flux residual $\left.\boldsymbol{\sigma}_{h, p}\right|_{\partial \kappa \backslash \Gamma}=\left.\left(\mathbf{B}(\mathbf{n}) \mathbf{u}_{\mathrm{DG}}^{+}-\mathcal{H}\left(\mathbf{u}_{\mathrm{DG}}^{+}, \mathbf{u}_{\mathrm{DG}}^{-}, \mathbf{n}\right)\right)\right|_{\partial \kappa \backslash \Gamma}$. With these definitions we deduce from (3.2) the following relationship between the residuals:

$$
B_{\mathrm{DG}}\left(\mathbf{u}-\mathbf{u}_{\mathrm{DG}}, \mathbf{v}\right) \equiv \sum_{\kappa \in \mathcal{T}_{h}}\left(\mathbf{r}_{h, p}, \mathbf{v}\right)_{\kappa}+\sum_{\kappa \in \mathcal{T}_{h}}\left(\boldsymbol{\sigma}_{h, p}, \mathbf{v}^{+}\right)_{\partial \kappa \backslash \Gamma}+\sum_{\kappa \in \mathcal{T}_{h}}\left(\boldsymbol{\rho}_{h, p}, \mathbf{v}^{+}\right)_{\partial \kappa \cap \Gamma}=0
$$


for all $\mathbf{v}$ in $S^{\mathbf{p}}\left(\Omega, \mathcal{T}_{h}, \mathbf{F}\right)$. The identity (4.2) is referred to as the Galerkin orthogonality property of the $h p-D G F E M$. On choosing $\mathbf{w}=\mathbf{u}-\mathbf{u}_{\mathrm{DG}}$ in (4.1), the linearity of $J(\cdot)$ and (4.2) yield the (primal) error representation formula:

$$
\begin{aligned}
J(\mathbf{u})-J\left(\mathbf{u}_{\mathrm{DG}}\right) & =J\left(\mathbf{u}-\mathbf{u}_{\mathrm{DG}}\right)=B_{\mathrm{DG}}\left(\mathbf{u}-\mathbf{u}_{\mathrm{DG}}, \mathbf{z}\right)=B_{\mathrm{DG}}\left(\mathbf{u}-\mathbf{u}_{\mathrm{DG}}, \mathbf{z}-\mathbf{z}_{h, p}\right) \\
& \equiv \mathcal{E}_{\Omega}\left(\mathbf{u}_{\mathrm{DG}}, h, \mathbf{p}, \mathbf{z}-\mathbf{z}_{h p}\right)=\sum_{\kappa \in \mathcal{T}_{h}} \eta_{\kappa}
\end{aligned}
$$

where

$$
\eta_{\kappa}=\left(\mathbf{r}_{h, p}, \mathbf{z}-\mathbf{z}_{h, p}\right)_{\kappa}+\left(\boldsymbol{\sigma}_{h, p},\left(\mathbf{z}-\mathbf{z}_{h, p}\right)^{+}\right)_{\partial \kappa \backslash \Gamma}+\left(\boldsymbol{\rho}_{h, p},\left(\mathbf{z}-\mathbf{z}_{h, p}\right)^{+}\right)_{\partial \kappa \cap \Gamma} \cdot
$$

We consider the following measurement problem: given a linear functional $J(\cdot)$ and a positive tolerance TOL, compute $J\left(\mathbf{u}_{\mathrm{DG}}\right) \in \mathbb{R}$ such that

$$
\left|J(\mathbf{u})-J\left(\mathbf{u}_{\mathrm{DG}}\right)\right| \leq \text { TOL } .
$$

A necessary and sufficient condition for this to hold is that the stopping criterion

$$
\left|\mathcal{E}_{\Omega}\left(\mathbf{u}_{\mathrm{DG}}, h, \mathbf{p}, \mathbf{z}-\mathbf{z}_{h, p}\right)\right| \leq \mathrm{TOL}
$$

is satisfied, cf. equation (4.3) . Unfortunately, the left-hand side of (4.6) involves the unknown analytical solution $\mathbf{z}$ to the dual problem (4.1). Let us therefore suppose that problem (4.1) has been solved numerically, using an $h p$-adaptive DGFEM for example, on a sequence of "dual" finite element spaces $S^{\tilde{\mathbf{p}}}(\Omega, \tilde{\mathcal{T}}, \tilde{\mathbf{F}})$, based on a "dual" partition $\tilde{\mathcal{T}}_{h}$ and "dual" polynomial vector $\tilde{\mathbf{p}}$, which may differ from the "primal" partitions $\mathcal{T}_{h}$ and "primal" polynomial vectors $\mathbf{p}$, respectively; in this case, we write $\tilde{\mathbf{z}}_{\mathrm{DG}}$ to denote the corresponding approximation to $\mathbf{z}$ from the finite element space $S^{\tilde{\mathbf{p}}}(\Omega, \tilde{\mathcal{T}}, \tilde{\mathbf{F}})$. We denote by $\mathbf{z}_{h}$ the $L_{2}(\Omega)$ projection of $\tilde{\mathbf{z}}_{\mathrm{DG}}$ on the primal finite element space $S^{\mathbf{p}}\left(\Omega, \mathcal{T}_{h}, \mathbf{F}\right)$ defined over the primal subdivision $\mathcal{T}_{h}$. Thereby, decomposing the error representation formula (4.3) into terms which are computable, namely those involving the numerical approximation $\tilde{\mathbf{z}}_{\mathrm{DG}}$ to the dual solution, and those that require the analytical dual solution $\mathbf{z}$, a sufficient condition for (4.6) is that

$$
\left|J(\mathbf{u})-J\left(\mathbf{u}_{\mathrm{DG}}\right)\right| \leq \mathcal{E}_{\mathrm{P}}+\mathcal{E}_{\mathrm{D}} \equiv \sum_{\kappa \in \mathcal{T}_{h}}\left|\tilde{\eta}_{\kappa}\right|+\left|\mathcal{E}_{\Omega}\left(\mathbf{u}_{\mathrm{DG}}, h, \mathbf{p}, \mathbf{z}-\tilde{\mathbf{z}}_{\mathrm{DG}}\right)\right| \leq \mathrm{TOL}
$$

where $\tilde{\eta}_{\kappa}$ is defined analogously to $\eta_{\kappa}$, cf. (4.4), with $\mathbf{z}$ replaced by $\tilde{\mathbf{z}}_{\mathrm{DG}}$. We emphasise here that the fundamental difference between the terms $\mathcal{E}_{\mathrm{P}}$ and $\mathcal{E}_{\mathrm{D}}$ is that in the former the absolute value signs appear under the summation over the elements $\kappa \in \mathcal{T}_{h}$, while in the latter the absolute value sign is outside the sum. It has been shown through numerical experiments in [8] (cf. also [5]) that $\mathcal{E}_{\mathrm{D}}$ is typically an order of magnitude smaller than $\mathcal{E}_{\mathrm{P}}$. Therefore, $\mathcal{E}_{\mathrm{D}}$ can be safely absorbed into $\mathcal{E}_{\mathrm{P}}$ without compromising the reliability of the adaptive algorithm when the stopping criterion (4.6) is replaced by $\mathcal{E}_{\mathrm{P}} \leq \mathrm{TOL}$. A bound of the form

$$
\left|J(\mathbf{u})-J\left(\mathbf{u}_{\mathrm{DG}}\right)\right| \lesssim \mathcal{E}_{\mathrm{P}} \equiv \sum_{\kappa \in \mathcal{T}_{h}}\left|\tilde{\eta}_{\kappa}\right|
$$

which explicitly involves the numerical solution $\tilde{\mathbf{z}}_{\mathrm{DG}}$ to the dual problem (through $\tilde{\eta}_{\kappa}$ defined above) is referred to as a Type I a posteriori error bound, cf. [8].

Remark 3 An alternative to the approach presented here would have been to completely eliminate the dual solution from the a posteriori error bound, through the use of the CauchySchwarz inequality on each of the terms appearing in $\eta_{\kappa}$, choosing $\mathbf{z}_{h, p}$ as the finite element 
interpolant (or quasi-interpolant) of $\mathbf{z}$ from $S^{\mathbf{p}}\left(\Omega, \mathcal{T}_{h}, \mathbf{F}\right)$, applying interpolation error estimates to bound $\left\|\mathbf{z}-\mathbf{z}_{h, p}\right\|_{L_{2}(\kappa)}$ and $\left\|\mathbf{z}-\mathbf{z}_{h, p}\right\|_{L_{2}(\partial \kappa)}$ in terms of powers of $h$ and $p$ and Sobolev seminorms of $\mathbf{z}$, and finally employing strong stability estimates of Sobolev seminorms of the dual solution in terms of Sobolev norms of the data for the dual problem. Hence, the resulting so-called Type II a posteriori error bound will not involve the dual solution. However, such an approach is not without practical drawbacks: quite apart from the fact that the derivation of a Type II a posteriori error bound requires a considerable amount of analytical work in order to establish strong stability results for the dual problem in various Sobolev norms and to determine the associated strong stability constants, typically, Type II bounds give pessimistic overestimates of the error. Consequently, when employed in an adaptive algorithm to approximate $J(\mathbf{u})$ by $J\left(\mathbf{u}_{\mathrm{DG}}\right)$ to within a prescribed tolerance, a Type II a posteriori error bound will lead to uneconomical meshes; see, [6].

For a user-defined tolerance TOL, we now consider the problem of designing an $h p$-finite element space $S^{\mathbf{p}}\left(\Omega, \mathcal{T}_{h}, \mathbf{F}\right)$ such that

$$
\left|J(\mathbf{u})-J\left(\mathbf{u}_{\mathrm{DG}}\right)\right| \leq \text { TOL }
$$

subject to the constraint that the total number of degrees of freedom in $S^{\mathbf{p}}\left(\Omega, \mathcal{T}_{h}, \mathbf{F}\right)$ is minimised. Following the discussion above we exploit the Type I a posteriori error bound (4.7) to construct $S^{\mathbf{p}}\left(\Omega, \mathcal{T}_{h}, \mathbf{F}\right)$ such that

$$
\mathcal{E}_{\mathrm{P}} \leq \mathrm{TOL}
$$

If the stopping criterion (4.10) is not satisfied, then the elements are marked for refinement/derefinement; to this end, we employ the fixed fraction mesh refinement algorithm (cf. [9]), with refinement and derefinement fractions set to $20 \%$ and $10 \%$, respectively.

Once an element $\kappa \in \mathcal{T}_{h}$ has been flagged for refinement or derefinement, a decision must be made whether the local mesh size $h_{\kappa}$ or the local degree $p_{\kappa}$ of the approximating polynomial should be adjusted accordingly. The choice to perform either $h$-refinement/derefinement or $p$-refinement/derefinement is dependent on the local smoothness of the primal and dual solutions $\mathbf{u}$ and $\mathbf{z}$, respectively.

Let us first consider the case when an element has been flagged for refinement. Clearly, if $\mathbf{u}$ or $\mathbf{z}$ are locally smooth, then $p$-refinement will be more effective than $h$-refinement, since the error will be expected to decay quickly within the current element $\kappa$ as $p_{\kappa}$ is increased. On the other hand, if $\mathbf{u}$ and $\mathbf{z}$ have low regularity within the element $\kappa$, then $h$-refinement will be performed. Thus, regions in the computational domain where the primal or dual solution are locally non-smooth are isolated from smooth regions, thereby reducing the influence of singularities/discontinuities as well as making $p$-refinement more effective. To ensure that the desired level of accuracy is achieved efficiently, in [8] an automatic procedure was developed for deciding when to $h$ - or $p$-refine, based on the smoothness-estimation strategy proposed by Ainsworth \& Senior [2] in the context of norm control for second-order elliptic problems.

If an element has been flagged for derefinement, then the strategy implemented here is to coarsen the mesh in low-error-regions where either the primal or dual solutions $\mathbf{u}$ and $\mathbf{z}$, respectively, are smooth and decrease the degree of the approximating polynomial in lowerror-regions when both $\mathbf{u}$ or $\mathbf{z}$ are not sufficiently regular, cf. [1] and [8].

Here, for computational simplicity, the dual finite element space $\tilde{S} \tilde{\mathbf{p}}(\Omega, \tilde{\mathcal{T}}, \tilde{\mathbf{F}})$ that is used to compute the discontinuous Galerkin approximation $\tilde{\mathbf{z}}_{\mathrm{DG}}$ to $\mathbf{z}$ will be constructed using the same mesh employed for $\mathbf{u}_{\mathrm{DG}}$, i.e. $\tilde{\mathcal{T}}_{h} \equiv \mathcal{T}_{h}$, with $\tilde{\mathbf{p}}=\mathbf{p}+\mathbf{1}$ (however, see [8], for the implementation of the general case when $\tilde{\mathcal{T}}_{h} \neq \mathcal{T}_{h}$ and $\tilde{\mathbf{p}}$ is unrelated to $\left.\mathbf{p}\right)$. 


\begin{tabular}{||c|c|c|c|c|c|c|c||}
\hline Nodes & Elements & DOF & $J\left(u-u_{\text {DG }}\right)$ & $\sum_{\kappa} \tilde{\eta}_{\kappa}$ & $\theta_{1}$ & $\sum_{\kappa}\left|\tilde{\eta}_{\kappa}\right|$ & $\theta_{2}$ \\
\hline 25 & 16 & 64 & $0.1207 \mathrm{E}-02$ & $0.1023 \mathrm{E}-02$ & 0.85 & $0.1938 \mathrm{E}-01$ & 16.06 \\
\hline 30 & 19 & 86 & $-0.8405 \mathrm{E}-02$ & $-0.8203 \mathrm{E}-02$ & 0.98 & $0.1006 \mathrm{E}-01$ & 1.20 \\
\hline 48 & 31 & 202 & $-0.6729 \mathrm{E}-02$ & $-0.6002 \mathrm{E}-02$ & 0.89 & $0.7279 \mathrm{E}-02$ & 1.08 \\
\hline 48 & 31 & 244 & $-0.1611 \mathrm{E}-02$ & $-0.1623 \mathrm{E}-02$ & 1.01 & $0.1927 \mathrm{E}-02$ & 1.20 \\
\hline 57 & 37 & 330 & $-0.9690 \mathrm{E}-03$ & $-0.9756 \mathrm{E}-03$ & 1.01 & $0.1043 \mathrm{E}-02$ & 1.08 \\
\hline 87 & 61 & 595 & $-0.8424 \mathrm{E}-03$ & $-0.8581 \mathrm{E}-03$ & 1.02 & $0.8654 \mathrm{E}-03$ & 1.03 \\
\hline 129 & 91 & 1078 & $-0.1075 \mathrm{E}-04$ & $-0.4017 \mathrm{E}-04$ & 3.74 & $0.4731 \mathrm{E}-04$ & 4.40 \\
\hline 139 & 100 & 1439 & $0.2691 \mathrm{E}-04$ & $0.2906 \mathrm{E}-04$ & 1.08 & $0.3580 \mathrm{E}-04$ & 1.33 \\
\hline 201 & 148 & 2490 & $-0.1456 \mathrm{E}-05$ & $-0.1290 \mathrm{E}-05$ & 0.89 & $0.2808 \mathrm{E}-05$ & 1.93 \\
\hline 263 & 199 & 3723 & $-0.4938 \mathrm{E}-06$ & $-0.6040 \mathrm{E}-06$ & 1.22 & $0.6721 \mathrm{E}-06$ & 1.36 \\
\hline 308 & 232 & 4876 & $-0.1196 \mathrm{E}-07$ & $-0.1123 \mathrm{E}-07$ & 0.94 & $0.4792 \mathrm{E}-07$ & 4.01 \\
\hline 383 & 292 & 6793 & $-0.5294 \mathrm{E}-08$ & $-0.5296 \mathrm{E}-08$ & 1.00 & $0.6621 \mathrm{E}-08$ & 1.25 \\
\hline 429 & 328 & 8548 & $-0.3450 \mathrm{E}-08$ & $-0.3457 \mathrm{E}-08$ & 1.00 & $0.4322 \mathrm{E}-08$ & 1.25 \\
\hline 542 & 418 & 12325 & $-0.1650 \mathrm{E}-09$ & $-0.1676 \mathrm{E}-09$ & 1.02 & $0.2047 \mathrm{E}-09$ & 1.24 \\
\hline
\end{tabular}

Table 1: Adaptive algorithm for the linear advection problem.

\section{$5 \quad$ Numerical experiments}

In this section we present some numerical experiments to demonstrate the performance of our $h p$-adaptive algorithm. In particular, we consider both linear and nonlinear hyperbolic problems; the extension of the a posteriori error analysis to nonlinear equations follows analogously, by constructing a suitable linearised dual problem, cf. [5].

\subsection{Linear advection}

In this example, we consider the scalar hyperbolic equation $\nabla \cdot(\mathbf{b} u)+c u=f$ on $\Omega=(0,1)^{2}$, where $\mathbf{b}=\left(10 y^{2}-12 x+1,1+y\right), c=-\nabla \cdot \mathbf{b}$ and $f=0$. The characteristics enter the square $\Omega$ across three of its edges: the two vertical sides and the bottom; they exit $\Omega$ through the top edge. We prescribe the boundary condition

$$
u(x, y)= \begin{cases}0 & \text { for } x=0,0.5<y \leq 1 \\ 1 & \text { for } x=0,0 \leq y \leq 0.5 \\ 1 & \text { for } 0 \leq x \leq 0.75, y=0 \\ 0 & \text { for } 0.75<x \leq 1, y=0 \\ \sin ^{2}(\pi y) & \text { for } x=1,0 \leq y \leq 1\end{cases}
$$

on the union $\Gamma_{-}$of the three inflow sides. Our aim is to compute the weighted normal flux through the outflow side $\Gamma_{+}$. Thus, we let $J(u)=\int_{\Gamma_{+}} \psi(x) u(x, 1) \mathrm{d} x$, where the weight function $\psi$ is defined by $\psi(x)=\sin (\pi x / 2)$ for $0 \leq x \leq 1$; thereby, the true value of the functional is $J(u)=0.246500283257585$, cf. [6].

In Table 1, we demonstrate the performance of the adaptive algorithm; here, we show the number of nodes, elements and degrees of freedom (DOF) in $S^{\mathbf{p}}\left(\Omega, \mathcal{T}_{h}, \mathbf{F}\right)$, the true error in the functional $J\left(u-u_{\mathrm{DG}}\right)$, the computed error representation formula $\tilde{\mathcal{E}}_{\Omega} \equiv \sum_{\kappa \in \mathcal{T}_{h}} \tilde{\eta}_{\kappa}$, the a posteriori error bound (4.8) and their respective effectivity indices $\theta_{1}$ and $\theta_{2}$. We see that initially, on very coarse meshes, the quality of the computed error representation formula $\tilde{\mathcal{E}}_{\Omega}$ is quite poor, in the sense that $\theta_{1}=\tilde{\mathcal{E}}_{\Omega} / J\left(u-u_{h}\right)$ is not close to one; however, as the mesh is refined the effectivity index $\theta_{1}$ approaches unity. Furthermore, we observe that the Type 


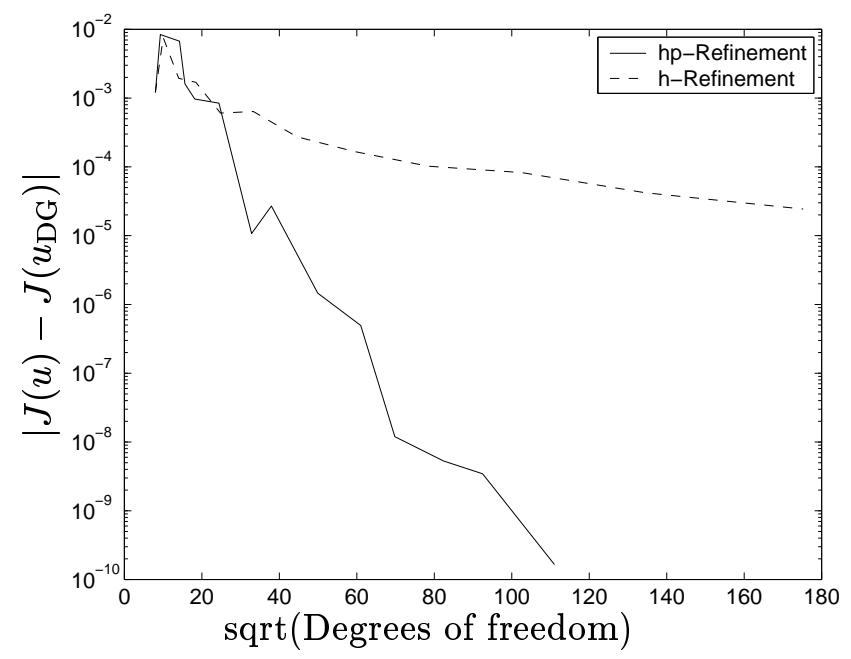

Figure 1: Comparison between $h-$ and $h p$-adaptive mesh refinement for the linear problem.

I a posteriori error bound is indeed sharp, in the sense that the second effectivity index $\theta_{2}$ overestimates the true error in the computed functional by a consistent factor as the finite element space $S^{\mathbf{p}}\left(\Omega, \mathcal{T}_{h}, \mathbf{F}\right)$ is enriched.

In Figure 1 we plot $\left|J(u)-J\left(u_{\mathrm{DG}}\right)\right|$, using both $h$ - and $h p$-refinement against the squareroot of the number of degrees of freedom on a linear-log scale. We see that after the initial transient, the error in the computed functional using $h p$-refinement becomes (on average) a straight line, thereby indicating exponential convergence of $J\left(u_{\mathrm{DG}}\right)$ to $J(u)$, despite the fact that $u$ is only piecewise continuous; this occurs since $z$ is a real analytic function on $\bar{\Omega}$. Figure 1 also demonstrates the superiority of the adaptive $h p$-refinement strategy over a traditional adaptive $h$-refinement algorithm. Indeed, on the final mesh the true error between $J(u)$ and $J\left(u_{\mathrm{DG}}\right)$ using $h p$-refinement is almost 6 orders of magnitude smaller than the corresponding quantity when $h$-refinement is employed alone.
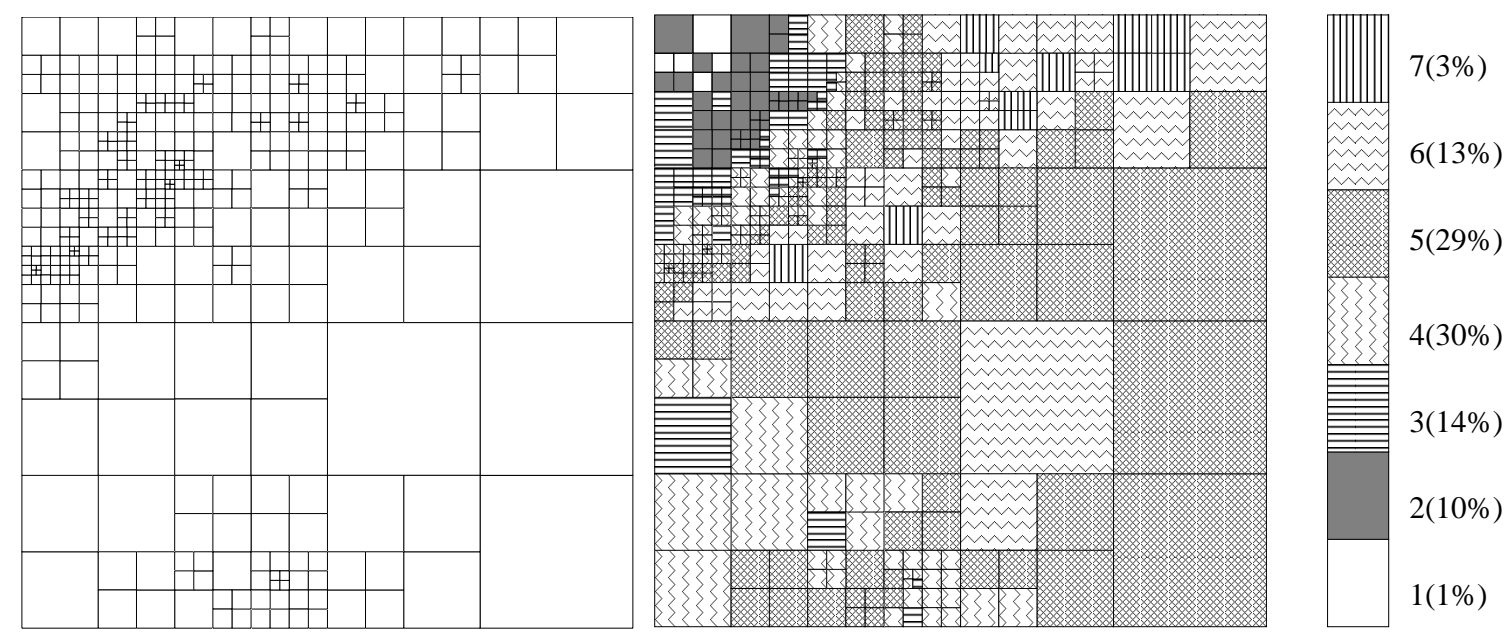

Figure 2: $h$ - and $h p$-meshes for the linear advection problem, with 542 nodes, 418 elements and 12325 degrees of freedom.

Finally, in Figure 2 we show the final primal mesh, after 13 adaptive mesh refinement 


\begin{tabular}{||c|c|c|c|c|c|c|c||}
\hline Nodes & Elements & DOF & $J\left(u-u_{h}\right)$ & $\sum_{\kappa} \tilde{\eta}_{\kappa}$ & $\theta_{1}$ & $\sum_{\kappa}\left|\tilde{\eta}_{\kappa}\right|$ & $\theta_{2}$ \\
\hline 12 & 12 & 36 & $0.2758 \mathrm{E}-01$ & -0.1613 & -5.85 & 0.4432 & 16.07 \\
\hline 19 & 25 & 75 & 0.1574 & 0.1175 & 0.75 & 0.2751 & 1.75 \\
\hline 35 & 53 & 159 & $0.9087 \mathrm{E}-01$ & $-0.3190 \mathrm{E}-01$ & -0.35 & 0.2244 & 2.47 \\
\hline 63 & 106 & 336 & $0.6027 \mathrm{E}-01$ & $0.6244 \mathrm{E}-01$ & 1.04 & 0.1215 & 2.02 \\
\hline 110 & 195 & 711 & $-0.1649 \mathrm{E}-01$ & $-0.1018 \mathrm{E}-01$ & 0.62 & $0.3127 \mathrm{E}-01$ & 1.90 \\
\hline 193 & 358 & 1400 & $-0.1802 \mathrm{E}-02$ & $-0.1831 \mathrm{E}-02$ & 1.02 & $0.7325 \mathrm{E}-02$ & 4.07 \\
\hline 327 & 623 & 2989 & $-0.2003 \mathrm{E}-03$ & $-0.1872 \mathrm{E}-03$ & 0.93 & $0.2077 \mathrm{E}-02$ & 10.37 \\
\hline 532 & 1032 & 5732 & $0.1064 \mathrm{E}-04$ & $0.1063 \mathrm{E}-04$ & 1.00 & $0.1773 \mathrm{E}-03$ & 16.66 \\
\hline 773 & 1512 & 10051 & $0.2939 \mathrm{E}-06$ & $0.3080 \mathrm{E}-06$ & 1.05 & $0.7398 \mathrm{E}-05$ & 25.18 \\
\hline 997 & 1958 & 15734 & $-0.2593 \mathrm{E}-07$ & $-0.2547 \mathrm{E}-07$ & 0.98 & $0.9037 \mathrm{E}-06$ & 34.85 \\
\hline 1311 & 2586 & 24710 & $0.3690 \mathrm{E}-08$ & $0.3691 \mathrm{E}-08$ & 1.00 & $0.3374 \mathrm{E}-07$ & 9.14 \\
\hline 1543 & 3049 & 33502 & $0.1710 \mathrm{E}-09$ & $0.1694 \mathrm{E}-09$ & 0.99 & $0.2581 \mathrm{E}-08$ & 15.09 \\
\hline 1857 & 3673 & 45653 & $-0.8103 \mathrm{E}-11$ & $-0.7714 \mathrm{E}-11$ & 0.95 & $0.3386 \mathrm{E}-09$ & 41.79 \\
\hline
\end{tabular}

Table 2: Adaptive algorithm for Burgers' problem.

steps. For clarity, we show the $h$-mesh alone, as well as the corresponding distribution of the polynomial degree and the percentage of elements with that degree. We see that the primal mesh has been predominantly $h$-refined in the neighbourhood of the discontinuity in $u$ emanating from $(x, y)=(0,0.5)$, with only a small number of elements being $h$-refined in the region where the second discontinuity enters $\Omega$ from $(x, y)=(0.5,0)$. Further, we see that $p$-refinement has taken place in the region to the right of the discontinuities in $u$, where both the primal and dual solutions are smooth; here, the polynomial degrees increase as as we move from $\Gamma_{-}$towards $\Gamma_{+}$, where the functional $J(\cdot)$ is specified.

\subsection{Burgers' equation}

Here, we consider the one-dimensional unsteady inviscid Burgers' equation for the scalar variable $\mathbf{u} \equiv u$; i.e., writing $x_{2}$ to denote time, we have $u_{x_{2}}+\left((1 / 2) u^{2}\right)_{x_{1}}=0$, on the (space-time) domain $\Omega=(0,3) \times(0,2)$, subject to the initial condition $u\left(x_{1}, 0\right)=2 /(1+$ $\left.x_{1}^{3}\right) \sin ^{2}\left(\pi x_{1}\right)$, and boundary condition $u\left(0, x_{2}\right)=0$, for $x_{2} \in[0,2]$. The analytical solution to this problem consists of three smooth 'hills' which form shock waves as time increases. These shocks will eventually merge to form a single line of discontinuity in the $\left(x_{1}, x_{2}\right)$ plane. Here, we only compute the numerical approximation up to a time after which the first two shock waves have merged, and select the functional of interest $J(\cdot)$ to be the value of the solution just before these two shocks interact with one another. More precisely, we choose $J(u)=u(1.95,1.35)$; thereby, the true value of the functional is $J(u)=0.451408206331223$.

In Table 2 we show the performance of our $h p$-adaptive finite element algorithm. As in the previous example, we see that the quality of the computed error representation formula is quite poor on coarse meshes; though, as the finite element space $S^{\mathbf{p}}\left(\Omega, \mathcal{T}_{h}, \mathbf{F}\right)$ is enriched the effectivity index $\theta_{1}$ tends to one. Furthermore, we again observe that the Type I a posteriori error bound (4.8) is asymptotically sharp, though the effectivity indices $\theta_{2}$ are now much larger than those computed for the linear advection problem. This is attributed to the loss of inter-element cancellation of the local error indicators $\tilde{\eta}_{\kappa}$, when the triangle inequality is employed en route to the Type I bound (4.8). This becomes evident in this example, as more $h$-refinement is required (cf. Figure 2 below) to get an accurate approximation of the functional of interest. 


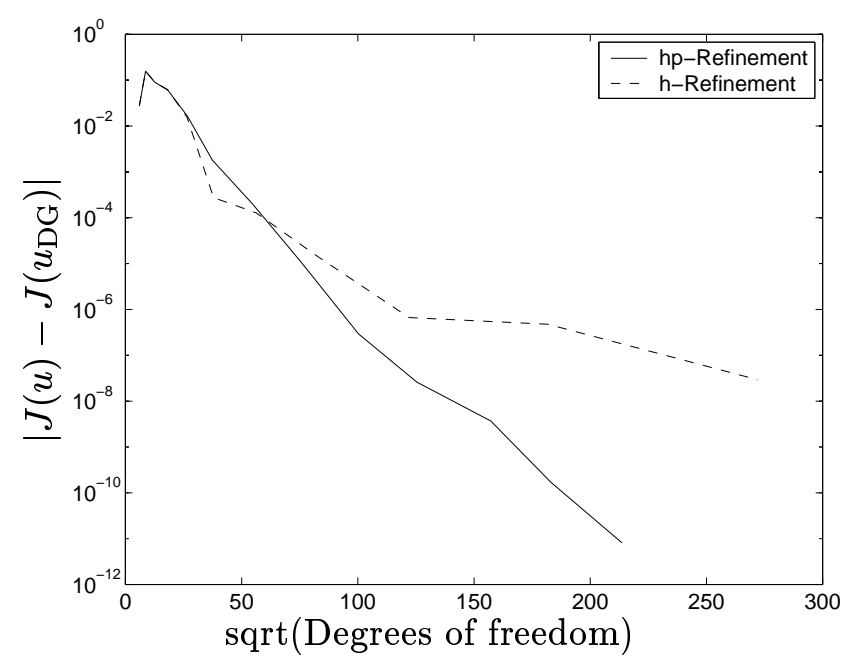

Figure 3: Comparison between $h$ - and $h p$-adaptive mesh refinement for Burgers' problem.

\begin{tabular}{||c|c|c|c|c|c|c|c||}
\hline Nodes & Elements & DOF & $J\left(u-u_{h}\right)$ & $\sum_{\kappa} \tilde{\eta}_{\kappa}$ & $\theta_{1}$ & $\sum_{\kappa}\left|\tilde{\eta}_{\kappa}\right|$ & $\theta_{2}$ \\
\hline 91 & 144 & 1728 & $-0.2995 \mathrm{E}-03$ & $-0.3024 \mathrm{E}-03$ & 1.01 & $0.2914 \mathrm{E}-02$ & 9.73 \\
\hline 129 & 219 & 3228 & $0.5143 \mathrm{E}-04$ & $0.4975 \mathrm{E}-04$ & 0.97 & $0.9527 \mathrm{E}-03$ & 18.52 \\
\hline 179 & 315 & 5312 & $-0.1884 \mathrm{E}-04$ & $-0.1885 \mathrm{E}-04$ & 1.00 & $0.2484 \mathrm{E}-03$ & 13.18 \\
\hline 245 & 445 & 8560 & $0.4813 \mathrm{E}-05$ & $0.4303 \mathrm{E}-05$ & 0.89 & $0.1164 \mathrm{E}-03$ & 24.19 \\
\hline 302 & 554 & 13480 & $-0.2541 \mathrm{E}-05$ & $-0.2662 \mathrm{E}-05$ & 1.05 & $0.4230 \mathrm{E}-04$ & 16.65 \\
\hline 352 & 650 & 17944 & $-0.1489 \mathrm{E}-05$ & $-0.1520 \mathrm{E}-05$ & 1.02 & $0.1824 \mathrm{E}-04$ & 12.25 \\
\hline 426 & 792 & 26260 & $-0.5522 \mathrm{E}-06$ & $-0.5662 \mathrm{E}-06$ & 1.03 & $0.5515 \mathrm{E}-05$ & 9.99 \\
\hline 487 & 912 & 35280 & $-0.2602 \mathrm{E}-07$ & $-0.2615 \mathrm{E}-07$ & 1.01 & $0.6618 \mathrm{E}-06$ & 25.44 \\
\hline 622 & 1171 & 51544 & $0.6738 \mathrm{E}-09$ & $0.6335 \mathrm{E}-09$ & 0.94 & $0.1119 \mathrm{E}-06$ & 166.02 \\
\hline
\end{tabular}

Table 3: Adaptive algorithm for Ringleb's flow.

In Figure 3 we compare the performance of the $h-$ and $h p$-mesh refinement algorithms for this problem. Again, we observe exponential convergence of the error in the computed functional using $h p$-refinement; on the linear-log scale, the convergence line is straight. Indeed, on the final mesh the true error between $J(u)$ and $J\left(u_{\mathrm{DG}}\right)$ using $h p$-refinement is almost 4 orders of magnitude smaller than the corresponding quantity when $h$-refinement is employed alone. Finally, in Figure 4 we show the final primal mesh after 12 adaptive mesh refinements. Here, we see that the $h$-mesh has been refined in the region upstream of the point of interest, thereby isolating the smooth region of $u$ from the two interacting shock waves; this renders the subsequent $p$-refinement in this region much more effective.

\subsection{Ringleb's flow}

In this final example we consider the steady two-dimensional compressible Euler equations; here, $\mathbf{u}$ represents the vector of conserved quantities $(\rho, \rho u, \rho v, \rho E)$, where $\rho,(u, v)$ and $E$ represent the density, Cartesian velocity and total energy per unit mass, respectively. Here, we consider Ringleb's flow for which an analytical solution may be obtained using the hodograph method. This problem represents a transonic flow which turns around an obstacle; the flow is mostly subsonic, with a small supersonic region around the nose of the obstacle. 

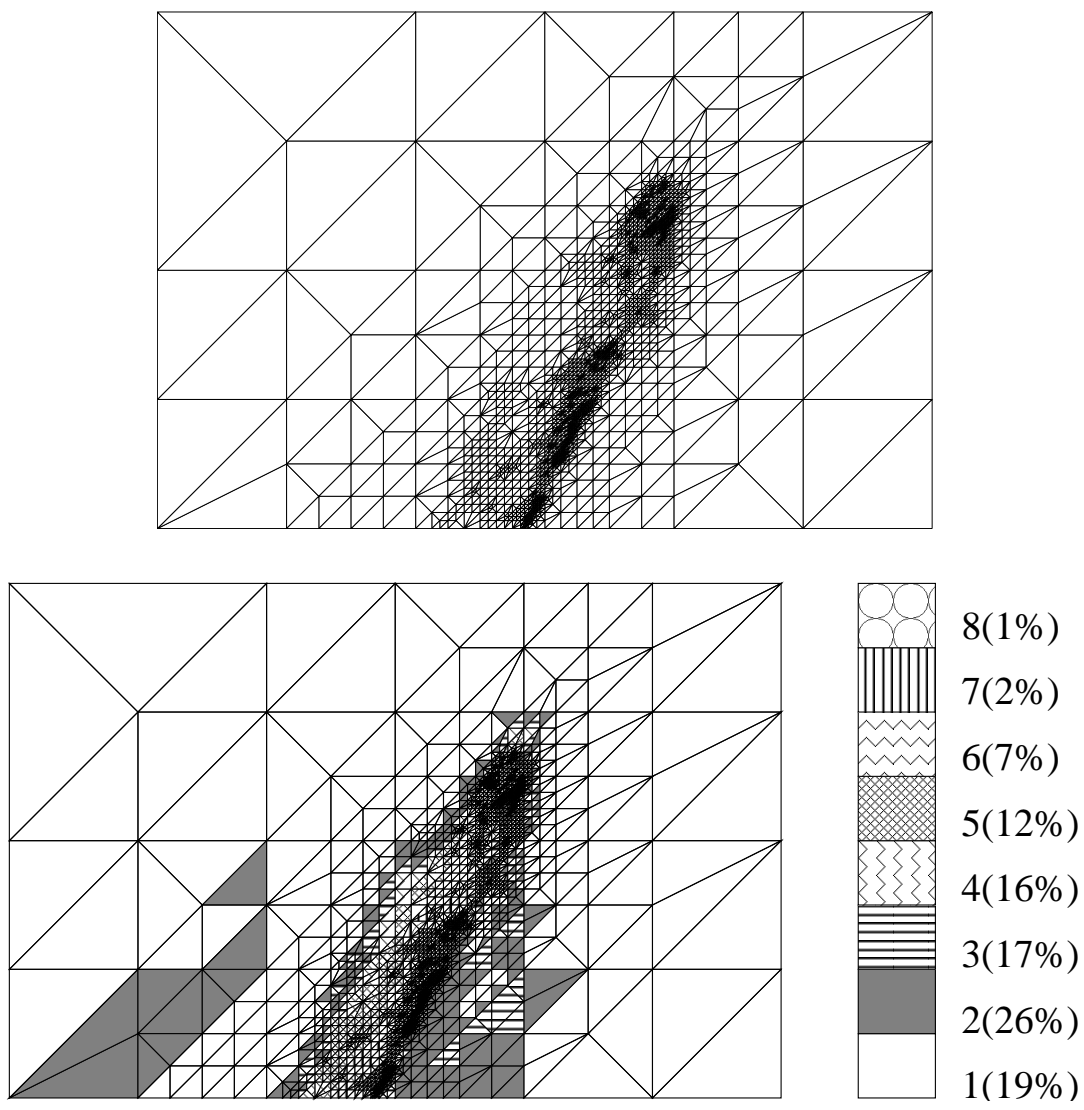

Figure 4: $h$ - and $h p$-meshes for Burgers' problem, with 1857 nodes, 3673 elements and 45653 degrees of freedom.

We take the functional of interest to be the value of the density at the point $(-0.4,2)$, i.e., $J(\mathbf{u})=\rho(-0.4,2)$; consequently the true value of the functional is given by $J(\mathbf{u})=$ 0.8616065996968034 . Table 3 shows the performance of our $h p$-adaptive algorithm; here we see that the quality of the computed error representation formula is extremely good, with $\theta_{1} \approx 1$ even on very coarse meshes. Furthermore, the Type I a posteriori error bound (4.8) overestimates the true error in the computed functional by about an order of magnitude, though there is a sharp increase on the last refinement. Figure 5 indicates exponential convergence for the error in the computed functional and again highlights the computational advantages of employing $h p$-mesh refinement when compared with the standard $h$-method.

\section{Conclusions}

Using duality arguments, in this paper we developed Type I a posteriori error bounds for the $h p$-version of the discontinuous Galerkin finite element method. The bounds have been implemented into an $h p$-adaptive finite element algorithm that is capable of delivering approximations to linear functionals of the solution to both linear and nonlinear hyperbolic problems, accurate to within a prescribed tolerance. The numerical experiments highlight the superiority of this general $h p$-adaptive strategy over a traditional $h$-refinement algorithm where the degree $p$ of the approximating polynomial is kept fixed at some low value. 


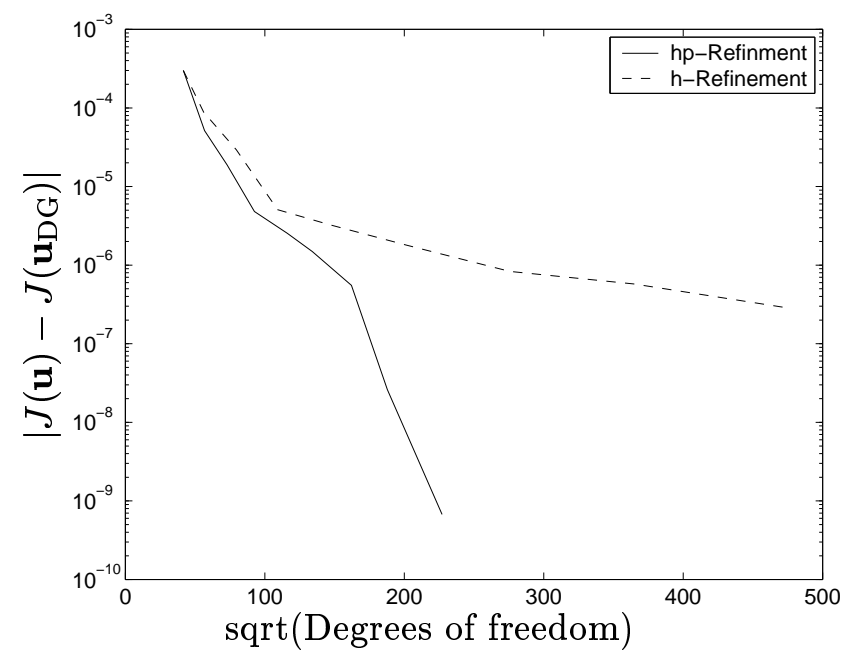

Figure 5: Comparison between $h-$ and $h p$-adaptive mesh refinement for Ringleb's flow.

\section{Acknowledgments}

Paul Houston and Bill Senior acknowledge the financial support of the EPSRC (Grant GR/N24230). The authors would also like to express their sincere gratitude to Professor Tim Barth for providing the code used to generate the analytical solution to Ringleb's flow.

\section{References}

[1] S. Adjerid, M. Aiffa, J.E. Flaherty, Computational methods for singularly perturbed systems. In J. Cronin and R.E. O'Malley, editors, Singular Perturbation Concepts of Differential Equations, AMS, Providence, 1998.

[2] M. Ainsworth AND B. Senior, An adaptive refinement strategy for $h p$-finite element computations. Appl. Numer. Maths. 26:165-178, 1998.

[3] K.S. BeY AND J.T. OdEN, hp-Version discontinuous Galerkin methods for hyperbolic conservation laws. Comput. Methods Appl. Mech. Engrg. 133:259-286, 1996.

[4] B. Cockburn, G.E. Karniadakis and C.-W. Shu. The development of discontinuous Galerkin methods. In: B. Cockburn, G. Karniadakis, and C.-W. Shu (Eds.) Discontinuous Galerkin Finite Element Methods. Lecture Notes in Computational Science and Engineering, Volume 11. Springer-Verlag 2000.

[5] R. Hartmann and P. Houston, Adaptive Discontinuous Galerkin Finite Element Methods for Nonlinear Hyperbolic Conservation Laws. In preparation.

[6] P. Houston, R. Rannacher And E. SÜLI, A posteriori error analysis for stabilised finite element approximations of transport problems. Comput. Meth. Appl. Mech. Engrg. 190(11-12):1483$1508,2000$.

[7] P. Houston, Ch. Schwab And E. Süli, Stabilized hp-finite element methods for first-order hyperbolic problems. SIAM J. Numer. Anal. 37(5):1618-1643, 2000.

[8] P. Houston And E. SüLI, hp-Adaptive Discontinuous Galerkin Finite Element Methods for Hyperbolic Problems. Numerical Analysis Group Research Report NA-01/05, University of Oxford, 2001. http://web.comlab.ox.ac.uk/oucl/publications/natr/na-01-05.html (Submitted).

[9] R. Rannacher, Adaptive finite element methods, in H. Bulgak and C. Zenger, eds., Proc. NATOSummer School Error Control and Adaptivity in Scientific Computing, Kluwer, Academic Publishers, 247-278, 1998. 